Topiques, études satoriennes

Topoï Studies, Journal of the SATOR

\title{
La figure quichottique et la réversibilité de la relation de mentorat dans Don Quichotte et Le Télémaque travesti
}

$\begin{array}{lllll}\mathbf{S} & \mathbf{A} & \mathbf{T} & \boldsymbol{\bullet} & \mathbf{R} \\ \mathbf{A} & R & E & P & O \\ \mathbf{T} & E & N & E & T \\ \boldsymbol{0} & P & E & R & A \\ \mathbf{R} & O & T & A & S\end{array}$

\section{Yen-Mai Tran-Gervat}

Volume 4, 2018

Maître-disciple : une relation topique

URI : https://id.erudit.org/iderudit/1074723ar

DOI : https://doi.org/10.7202/1074723ar

Aller au sommaire du numéro

Éditeur(s)

SATOR, Société d'Analyse de la Topique Romanesque d'Ancien Régime

ISSN

2369-4831 (numérique)

Découvrir la revue

Citer cet article

Tran-Gervat, Y.-M. (2018). La figure quichottique et la réversibilité de la relation de mentorat dans Don Quichotte et Le Télémaque travesti. Topiques, études satoriennes / Topoï Studies, Journal of the SATOR, 4, 1-13.

https://doi.org/10.7202/1074723ar
Résumé de l'article

Cet article étudie le traitement du couple mentor-disciple dans deux « romans de la folie romanesque ", Don Quichotte de Cervantès (1605-1615), le modèle de tous ces romans, et Le Télémaque travesti de Marivaux (1714), singulière parodie du Télémaque de Fénelon. Il s'efforce d'analyser les motifs comiques liés notamment à la transmission des savoirs livresques à un disciple ancré dans la culture populaire, mais aussi à l'éventuelle réversibilité de cette relation de mentorat. 


\section{La figure quichottique et la réversibilité de la relation de mentorat dans Don Quichotte de Cervantès et Le Télémaque travesti de Marivaux}

Le couple mentor-disciple n'est sans doute pas celui qui vient le plus immédiatement à l'esprit lorsqu'on envisage la question des duos comiques en littérature, que ce soit au théâtre ou dans le roman. Maître et esclave chez Plaute, puis maître et valet (ou leur équivalent, maîtresse et servante) de la commedia dell'arte à la comédie marivaudienne, en passant par la comedia espagnole et la comédie classique française ${ }^{1} .$. Ce rapport s'appuie le plus souvent sur la relation hiérarchique, tantôt confirmée par la sottise ou la couardise du valet (Clarin dans La nuit est un songe de Calderón), tantôt au contraire renversée par sa ruse supérieure (Scapin chez Molière), ou par l'extravagance ridicule du maître (Matamore dans L'Illusion comique de Corneille). Dans tous les cas, l'effet comique du couple de personnages suppose un contraste burlesque ${ }^{2}$ entre les deux figures, l'une admirable, l'autre ridicule, effet parfois redoublé par un décalage interne à chaque personnage entre son statut social et son discours.

Don Quichotte et Sancho Panza, dans le Don Quichotte de Cervantès (1610-1615) ${ }^{3}$, s'apparentent en partie à ces duos comiques traditionnels: écuyer improvisé, le paysan Sancho partage avec les valets de comédie un souci prioritaire de son confort physique et matériel ; à la fois naïf et inculte, représentant d'une certaine culture populaire ${ }^{4}$, il constitue l'interlocuteur idéal, du point de vue de la composition comique, pour son maître, noble de campagne nourri de livres de chevalerie jusqu'à s'en tourner l'esprit. La « folie par imitation

\footnotetext{
${ }^{1}$ Voir par exemple Catherine Dumas, Du gracioso au valet comique. Contribution à la comparaison de deux dramaturgies, 1610-1660, 2004.

2 J'emploie ici « burlesque » au sens large défini par Marmontel dans son article « Burlesque » de

l'Encyclopédie, repris dans les Éléments de littérature, dans Euvres complètes, tome V, p. 394-407 : « Le but moral de ce genre d'écrits est de faire voir que tous les objets ont deux faces; de déconcerter la vanité humaine, en présentant les plus grandes choses et les plus sérieuses d'un côté ridicule et bas; en prouvant à l'opinion qu'elle tient souvent à des formes. De ce contraste du grand au petit, continuellement opposés l'un à l'autre [je souligne], naît, pour les âmes susceptibles de l'impression du ridicule, un mouvement de surprise et de joie si vif, si soudain, si rapide, qu'il arrive souvent à l'homme le plus mélancolique d'en rire tout seul aux éclats [...] »

${ }^{3}$ Miguel de Cervantes, El ingenioso hidalgo don Quijote de la Mancha [1605-1615], edición del Instituto Cervantes, dirigida por Francisco Rico, con la colaboración de Joaquín Forradellas, 1998. Disponible en ligne sur le site du Centro Virtual Cervantes : https://cvc.cervantes.es/literatura/clasicos/quijote/default.htm ;

L'Ingénieux hidalgo don Quichotte de la Manche, tr. Jean Canavaggio et alii, 2010.

${ }^{4}$ Voir par exemple Maurice Molho, Cervantes : raices folklóricas, 1976, ou les lectures bakhtiniennes du chef d'œuvre de Cervantès développées dans Agustin Redondo, Otra manera de leer «El Quijote». Historia, tradiciones culturales y literatura, 1997.
} 
romanesque $^{5}$ » ou «principe quichottique ${ }^{6}$ » qu'invente Cervantès ajoute cependant à la relation entre maître et serviteur une dimension pédagogique : le lecteur fou se donne en effet non seulement pour mission de réaliser par ses actions les aventures romanesques décrites dans ses livres préférés, de donner voix par ses discours aux mots écrits sur le papier, mais également de convaincre les autres de leur pertinence et de la nécessité de s'y conformer et ce, non par une argumentation construite, mais par un discours d'autorité. La plupart des destinataires de ce discours sont incrédules, sceptiques, moqueurs ou inquiets ; Sancho, dont le premier acte de foi est son acceptation de partir à l'aventure contre la promesse d'une île à gouverner ${ }^{7}$, se place dans le même temps dans la position de l'ignorant qui a tout à apprendre (et, croit-il, à gagner) de ce nouveau maître, supérieur hiérarchique, mais surtout détenteur du savoir. Pour autant, maître et élève ne sont pas strictement un mentor et son disciple. J'ai exploré ailleurs ${ }^{8}$ la relation pédagogique maître-élève entre don Quichotte et Sancho Panza ; c'est ici la figure du «conseiller sage et expérimenté ${ }^{9} »$, instruisant et guidant son disciple dans ses actes et paroles, qui nous intéressera, non seulement dans le roman de Cervantès, mais dans un des « romans de la folie romanesque ${ }^{10}$ » qui ont pu s'en inspirer : un siècle après la publication du Quichotte en Espagne, puis en traduction partout en Europe, Marivaux rédigeait en effet Le Télémaque travesti de Marivaux (vers 1714), publié seulement en $1736^{11}$. Si je m'attache ici particulièrement à comparer cette œuvre avec Don Quichotte, c'est parce qu'elle présente la particularité de reposer à la fois sur une reprise du principe quichottique et sur celle de la figure de Mentor, via la « parodie mixte ${ }^{12} »$ du Télémaque de Fénelon.

\footnotetext{
${ }^{5}$ Michel Foucault, Histoire de la folie à l'âge classique, 1972, p. 47.

${ }^{6}$ Harry Levin, “The Quixotic Principle: Cervantes and other Novelists” dans Grounds for Comparison, 1972, p. 224-243.

${ }^{7}$ Cervantès, Don Quichotte, éd. cit., I, chap. VII.

${ }^{8}$ Yen-Mai Tran-Gervat, « Don Quichotte, maître de sérieux, modèle d'humour » dans C. François-Denève (dir.), La Chaire est triste : humour et enseignement, 2017, p. 85-95.

${ }^{9}$ Extrait de la définition de «mentor» donnée par le Trésor de la langue française informatisé, consulté le 29/01/18.

${ }^{10}$ C'est sous ce titre que j'ai étudié dans ma thèse de doctorat, «Le roman parodique au XVIII ${ }^{\mathrm{e}}$ siècle en Angleterre et en France » (Paris IV-Sorbonne, 2000), des œuvres comme : trois romans de jeunesse de Marivaux écrits dans les années 1713-1714 et réunis par F. Deloffre dans Euvres de jeunesse (Bibliothèque de la Pléiade, 1972), Le Télémaque travesti, «Le roman impromptu» de La voiture embourbée, Pharsamon ou les nouvelles folies romanesques; The Female Quixote de Charlotte Lennox (1752) ; Northanger Abbey de Jane Austen.

${ }_{11}$ Pierre de Marivaux, Le Télémaque travesti [1736], dans Euvres de jeunesse, éd. Frédéric Deloffre et Claude Rigaud, 1972.

12 Voir Gérard Genette, Palimpsestes. La littérature au second degré, 1982. Ce que G. Genette définit comme la parodie mixte, en prenant précisément ce roman de jeunesse de Marivaux comme exemple, repose sur une « homologie » entre hypertexte et hypotexte, « mais l'étage en-dessous »; ce que Marivaux lui-même, dans sa préface du Télémaque travesti, décrit ainsi : «on trouvera dans cette histoire même liaison et même suite d'aventures que dans le vrai Télémaque [de Fénelon].» (éd. cit., p. 722). Le principe est proche de celui du « travestissement burlesque », dont l'exemple type est le Virgile travesti de Scarron (un personnage noble est associé à un style trivial), mais se trouve conjugué avec le principe quichottique : ce n'est plus Enée qui parle comme un homme du peuple, mais un pseudo-Télémaque qui parle comme le paysan qu'il est.
} 
Le mentor quichottique est bien particulier : dans sa folie inaugurale, on le verra, il s'autoattribue la sagesse et l'expérience d'un mentor ; armé de son savoir romanesque et des règles d'action qui en découlent, il part dans le monde, et seul son disciple le suit pleinement dans cette aventure, mais celui-ci est doté, pour sa part, d'une expérience du monde que son maître ne possède pas, ouvrant la possibilité d'un renversement des rôles. Je me propose d'examiner les structures narratives ou discursives récurrentes caractéristiques de cette relation de mentorat et de sa réversibilité, dans le corpus limité choisi ici.

Le renversement de la relation de mentorat, qui fait du mentor un disciple est-elle symétrique? Autrement dit : dans ce renversement, le disciple qui se retrouve en position d'enseigner à son maître devient-il pour autant son mentor ? En examinant les modalités du renversement, et en particulier le point de vue de la narration lorsque celui-ci se produit, mais aussi l'évolution des personnages au fil du récit, à la faveur de ces occurrences, nous espérons préciser quelques éléments en termes de poétique et de topique du roman comique, mais aussi affiner la définition même du mentor.

\section{La mise en place d'un mentorat livresque (débuts de romans)}

Les débuts de romans permettent de voir la mise en place de la relation de mentorat selon des modalités prévisibles : le détenteur de l'ancienneté et du savoir (à défaut de la sagesse, dans des romans placés sous le signe de la folie) prend en charge la formation du sujet ignorant. Le topos est alors presque une définition, ou une tautologie : le mentor transmet son savoir à son disciple; la nuance propre au roman comique en général, et au roman quichottique en particulier, est que ce savoir est explicitement présenté comme ridicule, relevant de l'excès, en particulier exclusivement livresque, né d'une lecture déraisonnable, des livres de chevalerie ou du Télémaque de Fénelon dans le cas de notre corpus. Le topos pourrait alors être formulé ainsi : «Mentor comique_enseigner_savoir_livresque ${ }^{13}$ ».

Dans Don Quichotte, la mise en place de la relation de mentorat a lieu aux chapitres VII et VIII : les premiers chapitres du roman sont en effet focalisés sur le personnage de l'hidalgo vieillissant et oisif qui, dans sa folie, décide de partir en quête d'aventures chevaleresques sur les chemins d'Espagne. Mais ses premières interactions avec les autres personnages, jusqu'à l'introduction dans le récit du personnage de Sancho, sont de l'ordre de la censure (don Quichotte prétendant corriger les mauvaises perceptions des personnes qu'il rencontre, et

\footnotetext{
${ }^{13}$ Notons qu'un relevé topique complet inscrivant le corpus dans la sous-catégorie du roman quichottique (que j'ai appelé ailleurs le «roman de la folie romanesque ») devrait sans doute inclure un topos préalable comme Personnage_lire_à l'excès, qui est développé au chapitre I de Don Quichotte et dans le préambule du Télémaque travesti.
} 
inversement), plutôt que du conseil avisé d'un personnage sage et expérimenté à quelqu'un qu'il souhaite former. Certes, l'aubergiste qui «adoube » don Quichotte à sa demande, au chapitre III, donne des conseils pratiques bienvenus au pseudo-chevalier, mais cette rencontre est ponctuelle et la relation qui s'instaure n'est que temporaire : elle est déjà close au chapitre IV. Au contraire, lorsque don Quichotte décide, au chapitre VII, de s'adjoindre un écuyer pour sa seconde sortie, en la personne du laboureur Sancho Panza, la relation qui se noue alors ne s'interrompra qu'à la mort du maître, à la fin du second tome du roman de Cervantès. Bien sûr, le couple que forment ces deux personnages est d'abord comique, et en particulier burlesque, du fait notamment du contraste constant entre la culture livresque de l'un et le bon sens paysan du second: c'est ainsi qu'on se le représente le plus souvent. Mais on a également affaire dès le début à une relation entre un mentor en matière de chevalerie et son disciple désireux d'apprendre : l'âge, la connaissance, la figure d'autorité, appartiennent à don Quichotte, tandis que la naïveté, l'inexpérience chevaleresque et le respect du maître, sont du côté de Sancho. Même lorsque le savoir du maître déborde du champ romanesque, ses références restent livresques ${ }^{14}$. Dès la fin du chapitre VII de la première partie, au cours du tout premier trajet de leur longue histoire commune, don Quichotte instruit ainsi Sancho en matière de référents chevaleresques : «Tu dois savoir, ami Sancho, que ce fut une coutume fort usitée des anciens chevaliers errants, de faire leurs écuyers gouverneurs des isles ou des royaumes qu'ils conquéraient ${ }^{15}$. »

Les discours de don Quichotte, à partir de ce moment-là, malgré ses fréquents déboires, sont tous marqués par l'affirmation de son autorité détentrice de la « vérité » (énoncée au présent gnomique) et remplissent une fonction le plus souvent prescriptive, concernant les événements à venir, ou interprétative, concernant les actions passées. Ainsi, à l'issue de la fameuse aventure des moulins à vent :

[...] Les choses de la guerre sont, plus que toutes les autres, sujettes à de continuels changements ; d'autant que je pense, et c'est la vérité même, que le sage Freston, qui a volé mon cabinet et mes livres, a changé ces moulins en géants afin de m'ôter la gloire de les avoir vaincus, si grande est l'inimité qu'il me porte ; mais au bout du compte, ses méchants artifices ne pourront pas grand-chose contre la bonté de mon épée ${ }^{16}$.

\footnotetext{
14 On pense par exemple à la référence que fait don Quichotte à Ptolémée, au chapitre XXIX de la seconde partie, qui donne lieu à une plaisante confusion due à l'ignorance de Sancho.

${ }^{15}$ Cervantès, Don Quichotte, éd. cit., I, p. 136. « — Has de saber, amigo Sancho Panza, que fue costumbre muy usada de los caballeros andantes antiguos hacer gobernadores a sus escuderos de las ínsulas o reinos que ganaban ».

${ }^{16}$ Ibid., p. 139. « [...] las cosas de la guerra más que otras están sujetas a continua mudanza; cuanto más, que yo pienso, y es así verdad, que aquel sabio Frestón que me robó el aposento y los libros ha vuelto estos gigantes en molinos, por quitarme la gloria de su vencimiento: tal es la enemistad que me tiene; mas al cabo al cabo han de poder poco sus malas artes contra la bondad de mi espada. »
} 
On retrouve les mêmes caractéristiques dans Le Télémaque travesti, œuvre de jeunesse dans laquelle Marivaux lance sur les chemins d'Auvergne un jeune paysan, Timante Brideron, parti en quête de son père en compagnie de son oncle Phocion: tous deux sont des lecteurs passionnés du roman pédagogique de Fénelon, qu'ils connaissent par cœur. La particularité de l'entreprise de Marivaux est donc que le mentor qui a eu la tête tournée par les livres, Phocion, imite quasiment à la lettre le Mentor de Fénelon, calquant ses actions et ses discours sur ceux de son modèle : la structure choisie par Marivaux a donc cela de particulier que, là où la folie de don Quichotte est l'occasion, au-delà des situations comiques, d'une réflexion métafictionnelle, Le Télémaque travesti invente en quelque sorte un méta-mentor, à savoir une figure de mentor dont la référence n'est autre que Mentor lui-même. Une autre particularité est qu'alors que chez Cervantès, le disciple Sancho ne sait pas lire et qu'il ne connaît des livres de chevalerie que ce que son maître lui en dit, Brideron fils, également lecteur de Télémaque sur le conseil de son oncle, est lui aussi dans une démarche d'imitation livresque, comme en témoigne cette phrase qui résume l'effet de la lecture excessive chez le jeune homme : «L'enchantement fut complet, son oncle lui parut un Mentor ${ }^{17}$ ». Le terme « enchantement» qui renvoie à l'univers du merveilleux romanesque, désigne ici ironiquement la métamorphose qui n'existe qu'aux yeux du lecteur fou, sous l'effet d'un imaginaire trop intensément stimulé. C'est le même phénomène que celui expérimenté par don Quichotte dans le fameux épisode des moulins ${ }^{18}$, première «aventure » où Sancho l'accompagne, au terme de laquelle, placé devant l'évidence selon laquelle il a attaqué des moulins et non des géants, le pseudo-chevalier est prompt à accuser un " enchanteur ».

À l'inverse de Don Quichotte, cependant, chez Marivaux comme chez Fénelon, c'est le disciple, en l'occurrence le jeune homme, qui est le héros et personnage focal de l'histoire. Sa jeunesse et son tempérament fougueux l'emportent souvent sur la «sagesse » imitative que cherche à lui inculquer son oncle, qui a de ce fait des occasions de se retrouver dans la même situation que Don Quichotte à l'égard de Sancho, celle de devoir rappeler son élève à la règle de l'imitation héroïque : «Oh comme vous êtes bien loin de la sagesse du fameux Télémaque. De Pierrot à ce prince, quelle distance! De vous à Pierrot, il n'y a que le chemin de différence ${ }^{19}$. »

\footnotetext{
${ }^{17}$ Marivaux, Le Télémaque travesti, éd. cit., p. 725.

${ }^{18}$ Cervantès, Don Quichotte, éd. cit., I, p. 8.

${ }^{19}$ Marivaux, Le Télémaque travesti, éd. cit., p. 728.
} 
Chez Marivaux comme chez Cervantès, il est fréquent que le disciple résiste aux leçons du mentor. Cependant, dans Le Télémaque travesti, cette résistance est en quelque sorte programmée par l'hypotexte même, auquel la parodie marivaudienne emprunte ses situations et sa progression : si Télémaque apprenait sans difficulté, il n’y aurait ni histoire, ni leçon ; il en va de même pour l'intrigue burlesque qui en est l'homologue. Chez Cervantès, c'est le genre comique dans lequel s'inscrit le roman qui appelle la discordance entre les leçons du maître et la spontanéité populaire du disciple. En termes topiques, on a affaire dans les deux cas au même topos, qui pourrait se formuler en «Désaccord_disciple_mentor », mais dans un cas, c'est une étape indispensable du roman pédagogique, que l'on retrouverait tout aussi bien chez Fénelon, et dans l'autre, une caractéristique du roman comique, à travers la mise en œuvre du principe burlesque qui fait plaisamment contraster les ambitions livresques du mentor et la spontanéité populaire du disciple. Or le roman de Marivaux parodie un roman pédagogique, et convoque donc simultanément les deux programmes génériques.

Dès le début, dans les deux cas, l'ignorance du disciple ou sa tendance à douter des vertus de la référence livresque sont soulignées : c'est non seulement ce qui fait que le personnage «mentoré » a des choses à apprendre, mais aussi ce qui permet au principe opposé à la grandeur et à la noblesse représentées par les livres (le grotesque, le vulgaire, le populaire) de s'exprimer, d'autant plus librement que le roman comique, par définition, est son lieu naturel. Ainsi, au chapitre XVIII de la première partie de Don Quichotte, on trouve un de ces nombreux dialogues entre le maître et l'écuyer, après la mésaventure où Sancho s'est fait malmener sur une couverture par des plaisantins rencontrés dans une auberge :

[...] Et ce qui, selon mon faible entendement, serait meilleur et plus assuré, ce serait de nous en retourner à notre village, maintenant qu'est venu le temps de la moisson, et de nous occuper de nos biens, au lieu d'aller, comme on dit, de mal en pis et de fièvre en chaud mal.

- Comme tu t'y connais peu, Sancho, répondit don Quichotte, en matière de chevalerie ! Tais-toi et prends patience : un jour viendra où tu verras de tes yeux combien il est honorable d'exercer cette profession. $[\ldots]^{20}$.

Dans Le Télémaque travesti, pareillement, les situations sont nombreuses, où la réplique spontanée du disciple va à l'encontre des enseignements du maître: ainsi, au début des

\footnotetext{
${ }^{20}$ Cervantès, Don Quichotte I, éd. cit., p. 235. « [...] - Y lo que sería mejor y más acertado, según mi poco entendimiento, fuera el volvernos a nuestro lugar, ahora que es tiempo de la siega y de entender en la hacienda, dejándonos de andar de ceca en meca y de zoca en colodra, como dicen. I -iQué poco sabes, Sancho respondió don Quijote-, de achaque de caballería! Calla y ten paciencia, que día vendrá donde veas por vista de ojos cuán honrosa cosa es andar en este ejercicio. [...]»
} 
aventures de nos pseudo-Télémaque et Mentor, ceux-ci rencontrent Mélicerte, qui est leur Calypso, et Brideron entreprend de l'entretenir de son Ulysse de père :

[...] hélas, le grand homme voulut aller à la guerre, car le sang lui pétillait dans le corps comme la poudre dans le feu. Depuis ce temps, nos yeux ne l'ont point vu, nos mains ne l'ont point touché ; et peut-être un faquin de patron chez les Turcs, l'oblige à fumer ou à labourer la terre ; peut-être est-il enterré dans un fossé. Sa femme le pleure ; ses valets le pleurent, et nous le pleurons, mon oncle et moi. Si par hasard son chemin l'avait conduit ici, dites-nous-en ce que vous en savez, et ne méprisez pas son malheureux fils : car si vous étiez une malheureuse, vous seriez fâchée qu'on vous méprisât.

Phocion sourcilla un peu à ce discours; il lui avait paru trop commun. Peu s'en fallut qu'il ne recommençât une harangue, mais il craignit de blesser les règles de l'imitation: car en pareille occasion, Mentor ne desserra pas les dents ; il fallut donc se contenter de ce qu'on avait dit, et prendre le tout en patience ${ }^{21}$.

Le mentor comique de Marivaux est tenu de manière beaucoup plus stricte que don Quichotte aux « règles de l'imitation », du fait de l'hypotexte unique en jeu dans la parodie ${ }^{22}$, là où Cervantès convoque plutôt pour son lecteur fou un « hypogenre ", qui laisse à l'imitateur une certaine marge d'interprétation. Le comique burlesque chez Marivaux se joue donc aussi dans la relation à distance entre le texte que nous lisons et celui de Fénelon, alors que don Quichotte résume ses modèles chevaleresques et concentre ainsi dans ses discours à la fois l'exigence d'imitation et la référence de cette imitation.

Les prises de parole des disciples sont une des sources principales du comique, au point qu'elles tendent à devenir quantitativement plus importantes que celle des maîtres, constituant alors autant d'occasions de retournement de la relation mentor-disciple dans ces romans. Certes, la rigidité imitative du fou romanesque constitue un ressort comique à part entière, qui correspond au processus identifié par Bergson, «du mécanique plaqué sur du vivant ${ }^{23}$ » et porte avec soi une dimension satirique non-négligeable à l'égard des livres considérés, ou des excès mêmes de la lecture : c'est cet aspect qui justifie l'interprétation satirique de Don Quichotte, autorisée notamment par l'avant-propos parodique du roman. Mais le principe pourrait rester lui-même très mécanique et répétitif, donc lassant, si le roman comique ne devenait rapidement le lieu où « le vivant» (la nature, la vivacité d'esprit des personnages et du narrateur, la culture populaire) se met à déborder de toutes parts au point de constituer finalement ce que le fou se doit d'apprendre ou d'accepter pour compléter sa culture livresque et faire bonne figure dans le monde. Il ne s'agit pas ici des cruelles leçons de réalité que subit

\footnotetext{
${ }^{21}$ Marivaux, Le Télémaque travesti, éd. cit., p. 732.

${ }^{22}$ Pour la définition sous-jacente de la parodie en œuvre ici, voir : Yen-Mai Tran-Gervat, « Pour une définition opérationnelle de la parodie : parcours critique et enjeux d'un corpus spécifique », Narratologie, revue du Centre de Narratologie Appliquée de 1'Université de Nice-Sophia Antipolis, n 4, 2001, p. 65-78. Repris en ligne sur le site Revel@Nice, Cahiers de narratologie n 13, 2006 : http://revel.unice.fr/cnarra/document.html?id=358.

${ }^{23}$ Henri Bergson, Le Rirem 1900, p. 38.
} 
régulièrement don Quichotte (surtout dans le premier tome), mais, toujours au sein de la relation privilégiée avec Sancho, de l'ouverture de son esprit étroit ${ }^{24}$, quoique noble, au discours et à l'expérience de l'autre qu'est son disciple, dans une relation inversée.

\section{Formes et occurrences du renversement mentor-disciple}

Dans Don Quichotte, le mouvement est observable à différents moments du roman. Les occurrences les plus ironiques de ce retournement est atteint quand le disciple en vient à faire la leçon en matières livresques à son propre mentor, selon le principe de l'arroseur arrosé : c'est le cas par exemple au début de la seconde partie (1615), quand Sancho utilise les propres moyens de son maître pour justifier le décalage entre la réalité triviale et l'interprétation romanesque qu'il en donne, en faisant passer une paysanne, identifiée comme telle par don Quichotte, pour Dulcinée, sous prétexte d'un enchantement (chapitre X de la seconde partie). Je propose cependant de m'arrêter sur le cas remarquable du chapitre $\mathrm{XX}$ de la première partie, extrêmement riche et dense en la matière, qui permettra de mettre en évidence deux topoï distincts touchant à l'inversion de la relation mentor-disciple.

Le premier pourrait se formuler ainsi : «Disciple comique_défendre_références populaires » (topos a) ; le second inclut l'ironie supplémentaire dont nous traitions à l'instant : «Disciple comique_maîtriser_savoir livresque »(topos b).

Le chapitre XX se construit assez nettement en trois parties et constitue un point déterminant dans la première partie de Don Quichotte, car c'est là que le récit touche consciemment aux limites de la structure répétitive initiée au début du roman, à savoir: don Quichotte transforme une situation triviale en aventure chevaleresque, se heurte violemment à la réalité et en sort meurtri. Le retournement de la relation de mentorat accompagne ainsi une complexification du récit, qui se donne alors les moyens de continuer à explorer le principe de la folie romanesque sans pour autant tomber dans l'ennui de la répétition. Dans la première partie du chapitre, qui fait suite à une première initiative de Sancho qui s'avère involontairement conforme aux attentes livresques de son maître (l'inspiration du nom de «Chevalier de la Triste Figure »), don Quichotte continue à instruire Sancho, sur le ton d'autorité qui nous est à présent familier : «Ami Sancho, il faut que tu saches que je suis né par la volonté du ciel en cet âge de fer pour y faire revivre l'âge d'or ou doré, comme

\footnotetext{
${ }^{24}$ Le chapitre I, qui raconte la progression de la folie, précise qu'au moment où celle-ci s'installe définitivement, c'est à la faveur d'un « cerveau desséché » par l'excès de lectures chevaleresques.
} 
d'ordinaire on le nomme ${ }^{25}$ ». Il fait nuit noire et de grands coups se font entendre dans la nuit, qui stimulent l'imagination héroïque du pseudo-chevalier, mais éveillent une peur paralysante chez son écuyer, qui met alors tout en œuvre pour empêcher son maître d'aller explorer la source des bruits mystérieux : il entrave d'abord les pieds de Rossinante, puis à son maître impatient de pouvoir avancer dans l'aventure, promet une histoire qui lui permettra de patienter jusqu'au jour. Il se fait alors l'initiateur d'un type de récit que don Quichotte ne connaît pas, et se développe une occurrence remarquable de notre topos (a) : Sancho en effet, entreprend de raconter un conte qui commence comme un roman pastoral (une bergère poursuit un berger de ses assiduités), mais s'avère être un récit folklorique dont la fonction est d'aider l'auditeur à s'endormir, en comptant les chèvres que le berger doit faire passer une à une de l'autre côté d'une rivière avant de pouvoir échapper à sa poursuivante. C'est alors lui qui maîtrise la forme du récit et représente l'autorité en la matière, y compris lorsque don Quichotte proteste :

De la façon dont je la conte, répondit Sancho, se content dans mon pays toutes les fables. Je ne sais pas conter autrement, et il n'est pas bon, monsieur, que vous me demandiez de faire des modes nouvelles ${ }^{26}$.

Plus tard dans le chapitre, le jour étant arrivé, on découvre l'origine des bruits effrayants : il s'agit de moulins à eau dont les maillets en bois frappent l'eau. Le retournement est alors complet : Sancho parodie son maître, en reprenant au mot près les paroles prononcées par don Quichotte au début du chapitre, mais dans un contexte d'où tout danger est évacué, et dans une intention moqueuse ${ }^{27}$; se met alors en place le topos (b), puisque ce faisant, Sancho fait la preuve qu'il maîtrise à son tour le discours chevaleresque, fût-ce au sein d'une énonciation ironique. Celui-ci, cependant, s'articule immédiatement avec le topos (a), car tout ce qui précède et suit la reprise parodique de Sancho relève de l'initiation de don Quichotte à des référents triviaux, qu'il reconnaît comme tels, ainsi qu'à une réaction spontanée, par le rire, au ridicule de la situation. Plus qu'un renversement de la relation de mentorat, cet exemple met cependant en évidence une réciprocité de cette relation : la soudaine maitrise du référent chevaleresque par Sancho (topos b) témoigne de l'efficacité de l'enseignement de son maître ; de même que le rire de don Quichotte et l'absence d'hallucination hérö̈que de ce dernier

\footnotetext{
${ }^{25}$ Cervantès, Don Quichotte I, éd. cit., p. 259. «- Sancho amigo, has de saber que yo naci por querer del cielo en esta nuestra edad de hierro para resucitar en ella la de oro, o la dorada, como suele llamarse. »

${ }^{26}$ Ibid., p. 264. «— De la misma manera que yo lo cuento — respondió Sancho- se cuentan en mi tierra todas las consejas, y yo no sé contarlo de otra, ni es bien que vuestra merced me pida que haga usos nuevos. »

${ }^{27}$ Ibid, p. 272.
} 
témoigne de l'influence du disciple sur la folie du maître, qui est loin d'être schématique et prévisible.

L'examen des topoï a et b identifiés à la faveur de cet extrait exemplaire de Don Quichotte fait ressortir par contraste le fait que dans Le Télémaque travesti, on a davantage affaire à un cas particulier qu'à un cas typique de fiction quichottique.

Phocion remplit son rôle de Mentor en veillant à ce que son Télémaque de neveu ne se laisse pas séduire par des leurres qui le détourneraient de la sagesse et de sa quête et tâche difficilement — d'adapter son discours à la noblesse de son imitation. Brideron, de son côté, tout en ayant conscience de ces enjeux, se laisse librement aller à une parole spontanée, pleine d'expressions populaires: il imite les situations mais parle en paysan, se faisant alors régulièrement le mentor stylistique de son maître, si l'on en juge par la contagion comique qu'on constate alors dans le discours de Phocion. Ainsi, dans une situation similaire à celle où Mentor met en garde Télémaque contre la générosité trompeuse de Calypso ${ }^{28}$ :

[...] mais parlez donc; ne sommes-nous pas nés coiffés ? La bonne dame, que celle chez qui nous sommes ! sans elle nous étions bien bas percés, qu'en dites-vous ?

Ce que j'en dis, répondit Phocion? je dis que vous êtes un sot. Oh, oh ! repartit le neveu, quelle mouche vous pique? je n'aurais jamais deviné ce que vous dites. Oui, un sot, mon fils, ajouta Phocion ; laissezla faire, cette bonne dame, elle gâtera tout. Vous cherchez la sagesse et votre père ; que la peste m'étouffe, et vous aussi, si jamais vous trouvez l'un ou l'autre, en cas que vous croyez notre hôtesse. ${ }^{29}$

Cette contagion de la spontanéité du disciple au discours difficilement maintenu dans un registre noble par le pseudo-Mentor, constitue une justification diégétique de la spécificité de la « parodie mixte» marivaudienne. En effet, sans même avoir besoin de cette raison vraisemblable, Marivaux, lorsqu'il se donne un hypotexte unique et qu'il imite «l'étage en dessous », programme son récit pour une homogénéité du registre comique : quelle qu'en soit la raison (diégétique ou générique), le fait est que les tendances populaires qui contrastent avec le modèle héroïque apparaissent, dans les dialogues, aussi bien chez Phocion que chez Brideron. En ce sens, on n'a pas tant affaire à un Brideron qui serait le Sancho de PhocionQuichotte, qu'à deux personnages qui sont régulièrement leurs propres Sancho, à chaque fois que leur naturel ${ }^{30}$ prend le dessus sur l'imitation. Le roman pédagogique de Fénelon, que les

\footnotetext{
${ }^{28}$ Fénelon, Les Aventures de Télémaque, 1987, Livre I : « Mais quelle faveur du ciel nous a fait trouver, après notre naufrage, cette déesse ou cette mortelle qui nous comble de biens ? Craignez, repartit Mentor, qu'elle ne vous accable de maux; craignez ses trompeuses douceurs plus que les écueils qui ont brisé votre navire : le naufrage et la mort sont moins funestes que les plaisirs qui attaquent la vertu. Gardez-vous bien de croire ce qu'elle vous racontera. »

${ }^{29}$ Marivaux, Le Télémaque travesti, éd. cit., p. 736.

${ }^{30}$ Raccourci que nous employons ici pour désigner la représentation par Marivaux, dans un contexte « réaliste » au sens où il peut l'être au XVIII ${ }^{\mathrm{e}}$ siècle (voir par exemple Ian Watt, The Rise of the Novel, 1957), de la langue
} 
personnages ont en poche, joue alors le rôle de mentor de papier (ou de tuteur, si l'on veut employer un synonyme également valable pour les objets inanimés), chargé de maintenir dans le droit chemin des personnages tous deux régulièrement rattrapés par leur spontanéité de campagnards. Tantôt Phocion parvient à incarner cet impératif livresque et fait alors figure de mentor quichottique, tantôt il est emporté, comme son neveu, par l'énergie comique qui soustend tout le roman parodique de Marivaux.

Il y a cependant suffisamment d'occasions où Phocion s'efforce de jouer son rôle pour que le retournement porté par le topos a («Disciple comique_défendre_références populaires ») se produise et que Brideron revendique la vivacité populaire qui contredit l'enseignement livresque de Phocion. On en trouve une occurrence représentative au sein du récit de Brideron à Mélicerte :

\begin{abstract}
A peine achevait-il ces mots, qu'un rhumatisme de vingt ans vint prendre le métayer. Ahi! S'écria-t-il, mon mal me tourmente, guérissez-moi, mes bons enfants. Phocion avait un secret pour les rhumatismes, mais il détourne la tête, me faisant comprendre qu'autrefois Mentor avait bien combattu contre les barbares; mais non pas guéri de rhumatismes ; car voyez-vous, Madame, qui va à dia, ne doit pas tourner à huriau. Morguienne ! Lui-dis-je, guérissez ce bonhomme, personne ne le saura. Il le fit par le moyen de quelques herbes appliquées sur le mal ${ }^{31}$.
\end{abstract}

De même qu'au chapitre XX de Don Quichotte, dès avant l'aventure des moulins à eau, le maître a accepté un conseil de bon sens de son écuyer, l'autorisant à l'instruire en matière d'adaptation aux situations concrètes de l'existence ${ }^{32}$, de même ici on voit Phocion renoncer à l'imitation d'un modèle épique idéal pour aider son prochain : le disciple spontané, qui a tant à apprendre des livres, sait faire partager sa connaissance des situations quotidiennes à son maître.

Là encore, ce type d'occurrences narratives du retournement sont moins nombreuses dans Le Télémaque travesti que dans Don Quichotte, du fait du cas particulier dans lequel s'inscrit Marivaux, tenu par le principe d'imitation du fait même du travestissement burlesque ${ }^{33}$ constant qui caractérise ce roman. La contagion du style comique est partout, mais elle n'implique pas toujours un retournement : celui-ci n'a lieu que lorsque Phocion fait un effort conscient pour imiter la noblesse de son modèle, ce qui est finalement assez rare, comme le

populaire correspondant à la condition sociale des personnages. Cette notion de «naturel » vient rejoindre celle de «vivant» empruntée plus haut à Bergson, et s'oppose dans le roman comique et parodique au style noble imité de l'épopée et de ses avatars modernes en prose que sont les livres de chevalerie lus par don Quichotte (Amadis de Gaule notamment) et le roman pédagogique de Fénelon.

${ }^{31}$ Ibid., p. 746.

${ }^{32} \mathrm{Au}$ tout début du chapitre XX, Sancho recommande de s'arrêter pour se reposer et s'abreuver, et « ce conseil parut bon à don Quichotte » (éd. cit., p. 258). «Parecióle bien el consejo a don Quijote ».

${ }^{33}$ Voir ci-dessus, note 12. 
sont les entorses au schéma narratif ou discursif de Télémaque. Toute évolution de l'ignorance à la sagesse pour le pseudo-Télémaque suit celle du personnage de Fénelon, noncomique : la réversibilité de la relation de mentorat s'inscrit alors dans la structure même du roman pédagogique.

Le couple don Quichotte-Sancho et son équivalent marivaudien dans une œuvre par ailleurs très spécifique nous ont permis de mettre en évidence, dans un premier temps, un topos commun au roman quichottique et au roman pédagogique : «Mentor_enseigner_savoir livresque ». La dimension comique de cet enseignement repose sur l'excès et le systématisme lié à la «folie par imitation romanesque » : satirique et burlesque, ce mentorat comique met en évidence le contraste et l'inadéquation de ces livres pris au pied de la lettre avec le bon sens populaire et la naïveté incarnés par le disciple. Lorsque la relation se renverse, le disciple donne des leçons de vie et de spontanéité à son maître; nous avons alors identifié un topos comique («Disciple_défendre_référents populaires») et un topos ironique («Disciple_maîtriser_savoir livresque »). Mais il n'est pas certain qu'alors le disciple devienne pour autant à son tour un mentor. Ce statut en effet doit être à la fois revendiqué par le mentor et reconnu par le disciple : c'est le cas dans un sens, celui qui porte le discours livresque, mais non dans l'autre, du moins au niveau de la diégèse : aucun des disciples ne revendique le renversement de l'autorité ; celle-ci, telle une parenthèse carnavalesque, se produit ponctuellement, bien que régulièrement, puis chacun retrouve son rôle de départ. Le genre comique, certes, met l'accent sur la vie, la liberté de parole et la spontanéité des actions, par opposition au sérieux des genres nobles, mais sans doute cela dépasse-t-il le cadre de la relation de mentorat, pour signaler une relation complexe entre auteur et lecteur, qui relève sans doute plus du plaisir que du discours pédagogique.

Yen-Mai Tran Gervat

Université Sorbonne Nouvelle-Paris 3

\section{BIBLIOGRAPHIE}

BERGSON, Henri, Le Rire [1900], Paris, P.U.F., (Quadrige), 1985.

MIGUEL DE CERVANTES, El ingenioso hidalgo don Quijote de la Mancha [1605-1615], edición del Instituto Cervantes, dirigida por Francisco Rico, con la colaboración de Joaquín Forradellas, Barcelone, Institut Cervantes, 1998. [Francisco Rico (dir.)] 
MIGUEL DE CERVANTES, L'Ingénieux hidalgo don Quichotte de la Manche, Paris, Gallimard [2001] (Folio), 2010. [Jean Canavaggio et alii (trad.)]

DUMAS, Catherine, Du gracioso au valet comique. Contribution à la comparaison de deux dramaturgies, 1610-1660, Paris, Champion, 2004.

FENELON, François de Salignac de la Mothe, Les Aventures de Télémaque [composé en 1694 ; 1ère édition authentique, posth. : 1717] Paris, Classiques Garnier, 1987. [Jeanne-Lydie Goré (éd.)]

FOUCAULT, Michel, Histoire de la folie à l'âge classique, Paris, Gallimard (NRF), 1972.

GENETTE, Gérard, Palimpsestes. La littérature au second degré, Paris, Seuil, (Points), 1982.

LEVIN, Harry, "The Quixotic Principle: Cervantes and other Novelists » dans Grounds for Comparison, Cambridge, Harvard University Press, 1972, p. 224-243.

MARIVAUX, Pierre de, Le Télémaque travesti [1736], dans CEuvres de jeunesse, Gallimard, (Bibliothèque de la Pléiade), 1972. [F. Deloffre et C. Rigaud (éds)]

MARMONTEL, Jean-François, «Burlesque» dans les Éléments de littérature, Euvres complètes, Paris, Née de la Rochelle, 1787, t. V, p. 394-407.

MOLHO, Maurice, Cervantes : raíces folklóricas, Madrid, Gredos, 1976.

REDONDO, Agustin, Otra manera de leer «El Quijote». Historia, tradiciones culturales y literatura, Madrid, edición Castalia, 1997.

TRAN-GERVAT, Yen-Mai, " Pour une définition opérationnelle de la parodie : parcours critique et enjeux d'un corpus spécifique », Narratologie, revue du Centre de Narratologie Appliquée de l'Université de Nice-Sophia Antipolis, 4, 2001, L'Intertextualité, p. 65-78.

TRAN-GERVAT, Yen-Mai, « Don Quichotte, maître de sérieux, modèle d'humour » dans C. François-Denève (dir.), La Chaire est triste : humour et enseignement, L'Harmattan, (Éthique de la création), 2017.

WATT, Ian, The Rise of the Novel. Studies in Defoe, Richardson and Fielding, Berkeley, University of California Press, 1957. 